\title{
Prediction of Falls Using a 3-m Zigzag Walk Test
}

\author{
Suzuka Masuda, RPT ${ }^{1 \%}$, Kazuo Suganuma, RPT ${ }^{2)}$, Chika Kaneko, RPT, MS ${ }^{3)}$, \\ Kazuo Hoshina, RPT ${ }^{4}$, Taeko Suzuki, RPT ${ }^{4)}$, Toru Serita, RPT, MS ${ }^{2}$, \\ RYOKO SAKAKIBARA, RPT, MS ${ }^{3)}$ \\ 1) Department of Rehabilitation, Yamadakinen Hospital: 2-20-1 Ishihara, Sumida-ku, Tokyo 130-0011, \\ Japan. TEL: +81 3-3624-1151 \\ 2) Department of Tokyo Physical Therapy, Faculty of Medical Science, Teikyo University of Science \\ 3) Department of Rehabilitation, Kurata Hospital \\ 4) Department of Rehabilitation, Honmoku Hospital
}

\begin{abstract}
Objective] This study investigated the applicability of a 3-m zigzag walk test for the prediction of falls and examined the relationships among fall history, the 3-m zigzag walk test, 10-m walk, and age. [Subjects] A total of 50 elderly individuals ( 23 males and 27 females) aged 65 and over, who were able to walk independently, were studied. [Methods] Four poles made of PET bottles were placed on a 3-m walkway in a straight line to create a zigzag course, and the time needed to walk it was measured. The best results on days 1 and 2 were adopted for the fall and no-fall groups, and intra-rater reproducibility was evaluated by calculating the intra-class correlation coefficient and performing the paired t-test. For comparison of the time needed to walk the zigzag between the 2 groups, the unpaired t-test was performed. The relationships between the times needed to walk the 3-m zigzag and $10 \mathrm{~m}$ and age were analyzed by calculating the correlation coefficient with fall history as the dependent variable, in multiple logistic regression analysis with the times needed to walk the 3-m zigzag and $10 \mathrm{~m}$ and age as independent variables. For the optimal classification of the fall and no-fall groups, cutoffs were calculated based on the ROC curve. [Results] The paired t-test results did not show differences between measurements, and the ICC was 0.97 in the fall, and 0.94 in the no-fall groups. The fall group needed significantly more time than the no-fall group to walk the 3-m zigzag. Further, the Pearson product-moment correlation coefficient revealed a significant correlation between the times needed to walk the $3-\mathrm{m}$ zigzag and $10 \mathrm{~m}$, while no correlation was observed between the time needed to walk the $3-\mathrm{m}$ zigzag and age $(\mathrm{r}=0.225)$. The time needed to walk the $3-\mathrm{m}$ zigzag was extracted as a factor associated with fall history in multiple logistic regression analysis, with an odds ratio of 0.377 . Its significance as a variable was $p<0.01$. In the Hosmer-Lemeshow test of the study model, the rate of discrimination between the predicted and actual values was $82.0 \%$. [Conclusion] The cutoff time to walk the $3-\mathrm{m}$ zigzag was estimated to be 10.5 seconds, suggesting that this model may be a valid index for the prediction of falls.

Key words: Falls, 3-m zigzag walk test, Elderly
\end{abstract}

(This article was submitted Feb. 5, 2013, and was accepted Apr. 10, 2013)

\section{INTRODUCTION}

Up to the present, falls by the elderly have been examined by various methods, and there have been a number of reports regarding their causes and prediction. Falls occur more frequently inside than outside buildings, and this tendency has been explained by the large number of steps and turns required in indoor environments.

Falls by the elderly not only involve serious injuries, such as femoral neck fractures, but also lead to a decline in their daily activities. Itoh et al. ${ }^{1)}$ compared individuals with and without fall history experiences after exercise intervention, and reported that no improvement in the QOL was observed in the former, while the motor function improved in both groups. Based on this finding, falls may be a negative factor for the elderly's independence, decreasing their QOL, in

${ }^{*}$ To whom correspondence should be addressed.

E-mail: suzuka22635@yahoo.co.jp addition to their physical functions. Further, the prevention of falls in those at high risk due to previous fractures may reduce medical costs.

In the field of physical therapy, the 10-m walk, timed up \& go (TUG), and functional reach tests are used to assess the risk of falls, and their results have been reported to be associated with fall history.

I order to accurately assess the risk of indoor falls, it is essential to reproduce an indoor environment with a large number of turns, as they are considered a leading cause of indoor falls. In addition to this, the weak points of the conventional tests, such as difficulty in performing assessment in small spaces, necessity of equipment, and complicated procedures, also need to be considered. Masuda et al. ${ }^{2)}$ examined the elderly's ADL abilities by measuring the time needed to walk a $10-\mathrm{m}$ zigzag, without investigating its relationship with falls. When conducting a 10 -m zigzag walk test, a course of $10 \mathrm{~m}$ or more with a width of approximately $3 \mathrm{~m}$ is needed, in addition to necessary equipment, such as 
obstacles and mat switches. In this respect, considering the difficulty of conducting 10 -m zigzag walk tests in facilities with limited space, including those providing visiting rehabilitation and day care services, we developed a 3-m zigzag walk test (3ZWT) for the present study by partly revising the $10-\mathrm{m}$ version.

This study investigated was to determine the applicability of the 3ZWT for the prediction of falls, and examined the relationships among the 3ZWT, 10-m walk time, age, and fall history.

\section{SUBJECTS AND METHODS}

\section{Subjects}

A total of 50 elderly individuals ( 23 males and 27 females) aged 65 and over using outpatient rehabilitation and day care services were classified into 2 groups based on their fall history within the past 1 year (Table 1), falls 25 (12 males and 13 females) and no falls 25 (11 males and 14 females). Individuals who were able to walk independently without a cane were included in both groups, and those with orthopedic or central nervous system diseases possibly influencing measurements were excluded. Before the initiation of the study, the participants were provided with sufficient explanation of the study outline and objective, with written documents specifying privacy protection and their absolute right to withdraw from the study at any time, before to obtaining their written consent in conformity with the Helsinki Declaration.

\section{Methods}

For the classification of the participants into the fall and no-fall groups, Gibson's definition of falls ${ }^{3)}$ was adopted: "Unintentional contact of part of the body, such as the knee, upper limb, buttocks, and lower back, with the ground or some lower level". Then, falls when walking were included, and those when ascending/descending stairs and riding/getting off a bicycle were excluded.

The time needed to walk the zigzag was measured on a 3-m walkway. The starting point and goal were marked with $50-\mathrm{cm}$-wide plastic tape. To create a zigzag, 4 poles were placed on the walkway, each of which was made of 2 PET bottles $(500 \mathrm{ml} \times 2)$ adhered to each other at their caps. To ensure their stability, the lower bottles were filled with water. The poles were placed at intervals of $60 \mathrm{~cm}$ from the starting point. In the measurement, the participants were instructed to walk at their fastest speed after a start signal, and the time needed to step over the goal line was recorded. The time was adopted when the poles fell down due to contact, and not adopted when they were stridden over; in the latter case, measurement was repeated. Before measurement, a rehearsal was performed to practice the 3ZWT and confirm the absence of danger, such as staggering. We call this method of measuring the time needed to walk the $3-\mathrm{m}$ zigzag the 3-m zigzag walk test .

To evaluate the intra-rater reproducibility of 3ZWT, one rater performed measurements on 2 days separated by an interval of 3 days or more. On both days, the measurement was repeated after an interval of 1 minute or more, and the
Table 1. Attributes of the Fall and No-Fall groups

\begin{tabular}{lccl}
\hline & Fall $(\mathrm{n}=25)$ & No-Fall $(\mathrm{n}=25)$ & \\
\hline Sex & 12 males and & 11 males and & \\
& 13 females & 14 females & \\
Age (years) & $72.9 \pm 5.9$ & $72.9 \pm 5.6$ & n.s \\
Height $(\mathrm{cm})$ & $153.7 \pm 8.0$ & $155.0 \pm 10.8$ & n.s \\
Weight $(\mathrm{kg})$ & $57.4 \pm 10.2$ & $56.1 \pm 9.4$ & n.s \\
\hline
\end{tabular}

Mean \pm SD

best result was adopted and rounded off to the second decimal place.

The maximum speed when walking $10 \mathrm{~m}$ was measured on a 14-m course with a 2-m runway before the start point and after the goal. The time need to walk $10 \mathrm{~m}$ was measured from foot-ground contact after the starting line to that after stepping over the goal line. The participants were instructed to walk as fast as possible. The measurement was repeated after an twice with an interval of 1 minute or more, and the best result was adopted and rounded off to the first decimal place.

In the statistical analysis, the normal distribution of the variables was tested by the Shapiro-Wilk test. Subsequently, the attributes of the fall and no-fall groups were analyzed by performing the unpaired t-test. Intra-rater reproducibility was evaluated by calculating the intra-class correlation coefficient (ICC) and performing the paired t-test for the times adopted in each group on both measurement days. For comparison of the time needed to walk between the 2 groups, the unpaired t-test was performed. Further, to examine the association with the times needed to walk the 3ZWT and $10 \mathrm{~m}$ and age, the correlation coefficients with fall history were calculated and multiple logistic regression analysis was performed with the times needed to walk 3ZWT and $10 \mathrm{~m}$ and age as independent variables and fall history as the dependent variable. The variables were selected by using a forward selection procedure based on the likelihood ratio test. For the optimal classification of the fall and nofall groups, cutoffs were calculated based on the Receiver Operation Characteristic (ROC) curve. PASW Statistics 18 (SPSS Japan) was used for the analysis, with a significance level of $>1 \%$ in all tests.

\section{RESULTS}

The Shapiro-Wilk test results showed that not all of the variables were a normally distributed. The difference between the attributes of the fall and no-fall groups was slight (Table 1). No differences were observed between the t-test results on the first and second days in the fall or no-fall group (Table 2). Further, the $\operatorname{ICC}(1,1)$ was 0.97 in the falling, and 0.94 in the non-falling group. On comparison of the time needed 3ZWT between the 2 groups, the falling group needed significantly more time than the non-falling group $(\mathrm{p}<0.01) \quad($ Table 3$)$. The Pearson product-moment correlation coefficient showed a significant correlation, with $\mathrm{r}=0.553$ between the times needed to walk the $3-\mathrm{m}$ zigzag and $10 \mathrm{~m}$, and $\mathrm{r}=0.520$ between the time needed to 
Table 2. The intra-rater reproducibility of $3 \mathrm{~m}$ zigzag walk time

\begin{tabular}{lccc}
\hline & DAY1 & DAY2 & \\
\hline Fall & $12.6 \pm 2.5$ & $12.6 \pm 2.6$ & n.s \\
No-Fall & $8.7 \pm 2.1$ & $8.8 \pm 2.0$ & n.s \\
\hline Mean \pm SD, $\mathrm{p}<0.01$ & &
\end{tabular}

Mean \pm SD, $\mathrm{p}<0.01$

Table 3. Comparison of the time needed to walk the zigzag (Z-time) by the Fall and No-Fall groups

\begin{tabular}{ccc}
\hline & Fall & Non-Fall \\
\hline Z-time (seconds) & $12.6 \pm 2.5$ & $8.7 \pm 2.1^{*}$ \\
\hline Mean $\pm \mathrm{SD},{ }^{*} \mathrm{p}<0.01$ & &
\end{tabular}

Table 4. ROC curve-based cutoffs

\begin{tabular}{lcc}
\hline & Fall & Non-Fall \\
\hline Less than 10.5 seconds & 4 & 20 \\
10.5 seconds or more & 21 & 5 \\
\hline
\end{tabular}

Sensitivity: $80.0 \%$; specificity: $84.0 \%$; cutoff: 10.5 seconds

walk $10 \mathrm{~m}$ and age $(\mathrm{p}<0.01)$; no correlation was observed between the time needed to walk the $3-\mathrm{m}$ zigzag and age $(\mathrm{r}=0.225, \mathrm{p}>0.01)$. In multiple logistic regression analysis, the time needed to walk the 3-m zigzag was extracted as a factor influencing fall history $\left(\chi^{2}\right.$ test for the study model: $\mathrm{p}<0.01)$. The odds ratio for the time needed to walk the $3-\mathrm{m}$ zigzag was 0.377 (95\% confidence interval: 0.218 to 0.652 ), with a significance as a variable of $p<0.01$. The HosmerLemeshow test showed a result of $\mathrm{p}=497$ for the study model, with a rate of discrimination between predicted and actual values of $82.0 \%$. Further, the ROC curve-based cutoff was 10.5 seconds, and the area under the curve was 0.904 . (Table 4, Fig. 1).

\section{DISCUSSION}

This study examined the applicability of 3ZWT results for the prediction of falls.

Considering that no differences were observed between the attributes of the fall and no-fall groups, there may have been no influence of attributes.

Similarly, no differences were observed between measurement results on days 1 and 2 in both the fall and nofall groups, with high ICCs (falling: 0.97; and non-falling: 0.94). This suggests excellent intra-rater reproducibility of the measurement of the time needed to walk the 3-m zigzag, confirming its applicability for evaluation.

In the comparison of the time needed to walk the $3-\mathrm{m}$ zigzag, the fall group needed more time than the no-fall group. In multiple logistic regression analysis, the time needed to walk the 3-m zigzag was shown to be associated with fall history. Further, the Hosmer-Lemeshow test results showed a good goodness-of-fit, with a discrimination rate of $82 \%$, suggesting that this model may be a valid index for the prediction of falls. The cutoff based on the ROC

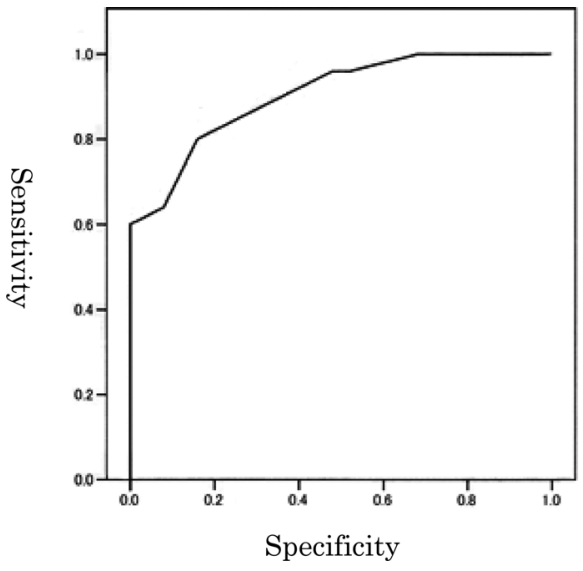

Fig. 1. ROC curve-based cutoffs $\mathrm{AUC}=0.904$

curve was 10.5 seconds (sensitivity: $80.0 \%$; and specificity: $84.0 \%$ ), the area under the ROC curve was large, 0.904 . Based on this result, the risk of falls was likely to increase when walking a 3ZWT course in 10.5 seconds or more.

The Pearson product-moment correlation revealed a significant correlation between the times needed to walk the 3-m zigzag and $10 \mathrm{~m}$, and between the time needed to walk $10 \mathrm{~m}$ and age, without showing such a correlation between the time needed to walk the 3-m zigzag and age. Based on this result, the time needed to walk the $3-\mathrm{m}$ zigzag may not be associated with the age.

Although the $10-\mathrm{m}$ zigzag test is used as an instrument to evaluate the elderly's ADL abilities at present, its applicability for the prediction of falls has not been reported, and the necessity of a walking course of $10 \mathrm{~m}$ or more when conducting it should be considered. To address these points, the $3 Z$ WT was developed by partly revising the $10-\mathrm{m}$ version to enable measurement in small spaces without using special devices. In recent years, an increasing number of predictors of falls have been reported. Arai et al. ${ }^{4)}$ calculated walking cycle fluctuations by measuring the time needed for a walk cycle using a compact accelerometer, and compared it with other motor indices. They showed that cycle fluctuations were shown to be the sole factor associated with falls and a valid predictor of falls in the elderly. However, it may be difficult to adopt this method on some occasions, as it requires equipment on measurement. Kobayashi et al. ${ }^{5)}$ devised a measurement instrumented which does not require equipment or techniques, called the Leg Opening and Closing While Sitting Test. They reported a high probability of fall prediction after opening and closing the legs 13 times, based on the ROC curve calculation. They also reported that this test showed good reproducibility, with an ICC of 0.984 in the fall, and 0.899 in the no-falls groups. Considering the high ICC, this test, as well as the 3ZWT, may be a valid predictor of falls. However, it is performed in a sitting position to ensure safety, and does not reproduce walking environment. Therefore, it is also necessary to examine the relationship between the obtained data and walking environments. Similarly, although the TUG test repro- 
duces a walking environment, it is possible for participants to voluntarily change the direction in this test. As difficult turns may be avoided. In the present study, a zigzag course was created with a number of turns in consideration of the necessity of reproducing a walking environment with left and right turns, as it has been reported that the majority of indoor falls by the elderly are associated with turns.

In the development of the 3ZWT, the appropriate material and height of poles, walking distance, and intervals between the poles were considered. To enable all raters to use this test, regardless of location, PET bottles of $500 \mathrm{ml}$ were used as a standardized, familiar material. We found that their height (approximately $25 \mathrm{~cm}$ ) was insufficient to prevent participants from striding over them, and ensure their stability was also an issue. As a solution, the caps of 2 PET bottles were stuck to each other to increase the height of the pole, and the lower bottle was filled with water to ensure its stability. The walking distance was limited to $3 \mathrm{~m}$ to allow measurement in small spaces. Regarding intervals between the poles, 50, 60, and $100 \mathrm{~cm}$ were considered. At intervals of $50 \mathrm{~cm}$, the walker's body frequently touched the poles. At intervals of $100 \mathrm{~cm}$, the course became linear with smaller turns. Based on this result, the poles were finally placed at intervals of $60 \mathrm{~cm}$ on a $3-\mathrm{m}$ course to provide a sufficient number of turns.

Some preceding epidemiological studies ${ }^{6}$ ) focusing on the physical factors of care dependency reported that impaired balance was the second leading cause of falls. Stalenhoef et al. ${ }^{7)}$ examined the main determinants for falls, such as the sex, age, number of previous falls, mental function, muscular strength, and balance, and reported that balance was the most closely associated with falls, with an odds ratio of 3.9. Considering that turns require high balance ability, the 3ZWT with a number of turns is likely to be a valid index $f$ or the prediction of falls.

As a study limitation, it may be necessary to consider recall bias, as this study was retrospectively conducted. In line with this, prospective studies may be necessary to continuously examine the study topic, focusing on the motor function which has been reported to be closely associated with the time needed to walk the 3ZWT zigzag, and it with other determinants of falls.

\section{REFERENCES}

1) Ito $Y$, Suganuma $K$, Serita $T$, et al.: Effects of a care prevention course on health-related qol and motor function according to history of falls. Phys Ther Sci, 2010, 25: 779-784.

2) Masuda K, Watanabe R, Mutou M, et al.: Activities of daily life in elderly, measuring the time needed to walk a 10-m zigzag. Japan Society of Physical Education, 2004, 55: 421.

3) Gibson MJ: Falls in later life. In: Improving the Health of Older People; A World View. New York: Oxford University Press, 1990, pp 296-315.

4) Arai $\mathrm{T}$, Shiba $\mathrm{Y}$, Watanabe $\mathrm{S}$, et al.: The relationship between the stride time variability, motor ability and fall in community-dwelling elderly people. Jpn Phys Ther Assoc, 2011, 38: 165-172.

5) Kobayashi K, Sato J: Prevention of fall using the sitting position legs open and close test. Jpn Phys Ther Assoc, 2009, 24: 77-80.

6) Graafmans WC, Otoms EM, Hofstee MH, et al.: Falls in the elderly: a prospective study of risk factors and risk profiles. Am J Epidemiol, 1996, 143: 1129-1136. [Medline] [CrossRef]

7) Stalenhoef PA, Diederiks JP, Knottnerus AJ: A risk model for the prediction of recurrent falls in community-dwelling elderly: a prospective cohort study. J Clin Epidemiol, 2002, 55: 1088-1094. [Medline] [CrossRef] 